\title{
Neuropathy after alcohol withdrawal
}

\author{
A. J. GARDNER ${ }^{1}$
}

From the Professorial Psychiatric Unit, St. George's Hospital, London

SUMMARY Three probable cases of neuropathy in alcoholics are described. They are very atypical for three reasons. First, each developed an acute asymmetrical neuropathy for the first time well after ceasing to drink, and after routine saturation with the vitamin B group. Secondly, none had previously suffered from neuropathy while still drinking. If this puzzling reversal of the usual order of clinical events has been noticed before, no record has been found in the literature. Further study by psychiatrists treating alcoholics aided by their neurological colleagues is suggested.

The relationship between peripheral neuropathy and alcoholism was first described by Lettsom in 1787 (Lettsom, 1792). Since then the occurrence of peripheral neuropathy during alcoholism, with the onset before abstinence and treatment, has been fully documented. The following report of three patients who do not fit this picture is presented to stimulate fresh thinking on an old subject.

\section{METHODS}

The patients were all inpatients and were part of a larger clinical and biochemical study of 51 alcoholics. The phenomena described were unexpected. Each had a full case history taken and physical examination by the ward doctor. In addition the author carried out an independent examination with the closest attention to the patients' neurological state and mental state within 72 hours of admission, and again immediately after the onset of neurological symptoms. In case 1 a consultant in neurology also examined the patient. There were no discrepancies between the findings on later comparison.

None of the three patients admitted taking any drugs before admission, therapeutically or otherwise, and none had suffered any previous neuropsychiatric illness. Alcohol was withdrawn abruptly and large doses of the B group vitamins were given parenterally, with the important exceptions of folic acid and vitamin B12. The only other drug given to the patients was phenytoin (Epanutin) $100 \mathrm{mg}$ t.d.s. for one week. At no time was disulfiram or any similar drug administered. The patients were nursed as inpatients during the period of investigation, and as the nursing staff were experienced and psychiatric-

1 Present address: Department of Psychological Medicine, The London Hospital, Whitechapel, E.1. ally trained, the possibility of the subjects having taken any alcohol after withdrawal was remote. In addition all three patients denied taking any more alcohol.

Many tests were carried out. The ones which might be most relevant in these cases were the serum $B_{12}$ and serum folate levels, which were estimated during the first 72 hours in cases 1 and 2 and at the onset of neuropathy in case 3 . The time limit was considered essential, as anticonvulsant medication for more than a brief period could affect the serum folic acid levels and in time the ward diet could well result in a slow replenishment of folic acid stores. The above estimations were carried out at the Atkinson Morley Hospital's laboratory, where the normal ranges are: serum $B_{12} 100-900 \mathrm{pg} / \mathrm{ml}$., and serum folic acid 4-16 $\mathrm{ng} / \mathrm{ml}$.

\section{CASE 1}

This 50 year old man had a history of heavy drinking for 20 years. He had been drinking on the day of admission, but apart from being partly drunk there were no abnormal signs.

After alcohol was withdrawn and the above regime was started, the patient developed the 'shakes' which lasted for three days. He was then well until the ninth day when he noticed the rapid onset of a burning pain in the right forearm and all the fingers of his hand.

Examination findings confirmed by a neurologist included tenderness on palpation of all muscle groups of the right forearm, weakness of these muscle groups, and of the right hand. The tendon reflexes of the right upper limb and both lower limbs were decreased. Sensory loss was found over all the right hand. There was no evidence of median, ulnar, or radial nerve damage nor of a cervical root lesion. A diagnosis of asymmetrical neuropathy was made. Other than giving him Becosym (which does not 
contain folic acid or vitamin $\mathbf{B}_{12}$ ), no other action was taken. Recovery took 11 weeks.

The results of investigations were as follows: Haemoglobin $14.0 \mathrm{~g} / 100 \mathrm{ml}$.; WBC 7,500/cu. mm; erythrocyte sedimentation rate (ESR) $8 \mathrm{~mm} / 1 \mathrm{hr}$; blood urea $26 \mathrm{mg} / 100 \mathrm{ml}$; t total protein-7.8 g/ $100 \mathrm{ml}$; electrophoresis normal; total bilirubin $0.3 \mathrm{mg} / 100 \mathrm{ml}$.; alkaline phosphatase 123 i.u./l.; SCOT 18 i.u./l.; SGPT 4 i.u./l.; WR negative. The urine contained neither albumin nor sugar and there were no porphyrins. Radiographs of the chest and skull were normal; serum $\mathbf{B}_{12}$ level was $300 \mathrm{pg} / \mathrm{ml}$.; serum folate $0.5 \mathrm{ng} / \mathrm{ml}$.

\section{CASE 2}

The patient was a 43 year old woman with a 23 year history of heavy drinking. She had not suffered any previous neuropsychiatric symptoms. On admission the findings and treatment regime were the same as in case 1 .

She suffered the 'shakes' for three days and was then well until the 15 th day when she developed tingling in the left hand and all the fingers of her left hand. The tingling soon changed to burning. The only abnormal finding was tenderness of the muscles of the left hand. Symptoms and signs subsided slowly over five days. The results of investigations were as follows: haemoglobin $12.0 \mathrm{~g} / 100 \mathrm{ml}$.; WBC $6,300 / \mathrm{cu}$. mm; ESR $6 \mathrm{~mm} / 1 \mathrm{hr}$; blood urea $18 \mathrm{mg} / 100 \mathrm{ml}$; total protein-6.4 g/100 ml.; electrophoresis was normal; total bilirubin $0.2 \mathrm{mg} /$ $100 \mathrm{ml}$.; alkaline phosphatase 60 i.u./l.; SGOT 13 i.u./l.; SGPT 2 i.u./l.; WR negative. The urine contained neither albumin nor sugar and there were no porphyrins present. The chest and skull radiographs were normal. Serum $B_{12}$ level was $190 \mathrm{pg} / \mathrm{ml}$. and serum folate $2.9 \mathrm{ng} / \mathrm{ml}$.

\section{CASE 3}

A male patient aged 51 years had a 33 year history of heavy drinking. Findings on admission and treatment were as for cases 1 and 2 .

He suffered the 'shakes' for two days then remained well until the 39th day, when he complained of burning in his calves, ankles, and feet. Abnormal neurological findings consisted of reduced right knee jerk and reduced appreciation of pin-prick and light touch over the lateral aspect of the left leg and medial surface of his right ankle. A diagnosis of asymmetrical neuropathy was made. This patient's serum folic acid and serum $B_{12}$ levels were not estimated within the first 72 hours, but were estimated at the onset of his neuropathy. Recovery took six weeks.

The results of investigations were as follows: Haemoglobin 13.8 g/100 ml.; WBC 7,800/cu. mm; ESR $9 \mathrm{~mm} / 1 \mathrm{hr}$; blood urea $30 \mathrm{mg} / 100 \mathrm{ml}$; ; total protein $7 \cdot 2 \mathrm{~g} / 100 \mathrm{ml}$; electrophoresis normal; total bilirubin $0.5 \mathrm{mg} / 100 \mathrm{ml}$; ; alkaline phosphatase 95 i.u./l.; SGOT 14 i.u./l.; SGPT 4 i.u./l.; WR negative. The urine contained neither albumin nor sugar and there were no porphyrins present. The chest and skull radiographs were normal. Serum $\mathbf{B}_{12}$ level was $150 \mathrm{pg} / \mathrm{ml}$. and serum folate $2.9 \mathrm{ng} / \mathrm{ml}$.

All three cases have the common finding of a normal serum $\mathrm{B}_{12}$ level associated with an abnormally low serum folate level.

\section{DISCUSSION}

Neuropathy as a complication of alcoholism must be known to all physicians. However, its presentation for the first time well after complete alcohol withdrawal seems to have gone unrecorded, though physicians have probably observed it. It follows that a study of this particular clinical variety of neuropathy and its possible relationship to folate status in alcoholism has yet to be published.

One explanation for the clinical events described above could be that the neuropathy was already present on admission and missed. This explanation can be ruled out with confidence, as not only was a full examination made $\stackrel{\mathbb{1}}{2}$ independently by two doctors, but one was particularly looking for neurological signs as part of a larger research project.

It would be valid to challenge the diagnosis of neuropathy but this still leaves the occurrence of neurological signs after alcohol withdrawal unexplained. Nerve conduction studies would have an important part in future investigations.

Deficiency of a B group vitamin other than folic acid is possible but improbable, as all three patients were saturated with $B$ group vitamins (except $\mathrm{B}_{12}$ and folic acid) before the onset of neuropathy. However, estimations of thiamine deficiency from red cell transketolase studies should be included in future inquiries. It is just possible that aneurine deficiency existing before vitamin saturation resulted in a pathological process, the clinical manifestations of which only appeared later. Vitamin $\mathrm{B}_{12}$ deficiency can almost certainly be excluded, in view of the normal serum $B_{12}$ levels.

The folic acid deficiency could be significant but the findings could as well be due to chance. The finding does, however, provide the only clue, though in itself an inadequate one, as a starting point for speculation.

The curious time sequence of clinical events remains the real puzzle. As this occurred in three 
cases out of 51 cases of alcoholism, it seems unlikely to be a rarity. The absence of similar cases from an otherwise voluminous literature on alcoholism complicated by neuropathy is another puzzle. Their possible relationship to the cases described by Gardner-Thorpe and Benjamin (1971) is an interesting one. They reported six cases of peripheral neuropathy occurring in abstinent alcoholics on disulfiram medication and they review a further 16 similar cases of peripheral neuropathy and six cases of optic atrophy previously recorded. The time of onset of peripheral neuropathy after alcohol withdrawal and commencement of disulfiram medication varied from 10 days to three years. Four of their cases made a fair or good recovery after stopping disulfiram. The three cases reported here developed their neuropathies on the ninth, 15th and 39th days after alcohol withdrawal. The chief point of similarity between the cases described by Gardner-Thorpe and Benjamin and the cases reported by the writer is that they are the only cases recorded in the literature where peripheral neuropathy presented after, not before, alcohol withdrawal.

The major point of difference is that GardnerThorpe and Benjamin's cases were all treated with disulfiram, whereas the cases described here were not given disulfiram at any time. This raises the possibility that disulfiram medication was not necessarily responsible for the development of peripheral neuropathy in all their cases. In fact, those cases presenting with neuropathy soon after alcohol withdrawal and the commencement of disulfiram therapy could be further examples of the cases presented here. It is suggested that in at least some of their cases, the relationship between peripheral neuropathy and disulfiram was coincidental.
One other point deserves comment. The only case of Gardner-Thorpe and Benjamin's in which the serum $B_{12}$ and serum folate were recorded demonstrated a normal serum $B_{12}$ level and an abnormally low serum folate level. This finding is a constant feature of the three cases recorded in the present paper.

\section{CONCLUSION}

Peripheral neuropathy in alcoholics can present for the first time after withdrawal of alcohol and in the absence of any toxic drug such as disulfiram. This phenomenon has not been recorded before. The aetiology is at present obscure. However, it can be said with some confidence that such cases are most likely to be observed by psychiatrists treating alcoholics. It might prove fruitful if interested psychiatrists discovering new cases investigated them with a neurological colleague. The results could increase our knowledge of the still incompletely solved relationship between peripheral neuropathy and alcoholism.

It is a pleasure to thank the staff of St. George's Hospital, London S.W.1 and Dr. M. Glatt of St. Bernard's Hospital for permission to study their patients. I am indebted to Professor A. Crisp for his constant encouragement and Professor G. F. M. Russell for his clear, constructive criticism.

\section{REFERENCES}

Gardner-Thorpe, C., and Benjamin, S. (1971). Peripheral neuropathy after disulfiram administration. Journal of Neurology, Neurosurgery, and Psychiatry, 34, 253-259.

Lettsom, J. C. (1792). Some remarks on the effects of lignum quassiae amarae. Memoirs of the Medical Society of London, 1, 128-165 (cf. pp. 158-161). 